\title{
Maser polarization with ALMA
}

\author{
Andrés F. Pérez-Sánchez ${ }^{1}$ and Wouter Vlemmings ${ }^{2}$ \\ ${ }^{1}$ Argelander Institute for Astronomy, University of Bonn \\ D-53121, Bonn, Germany \\ email: aperez@astro.uni-bonn.de \\ ${ }^{2}$ Chalmers University of Technology, Onsala Space Observatory \\ SE-439 92 Onsala, Sweden.
}

\begin{abstract}
Once ALMA full polarization capabilities are offered, (sub-)mm polarization studies will enter a new era. It will become possible to perform detailed studies of polarized maser emission towards for example massive star forming regions and late-type stars such as (post-) Asymptotic Giant Branch stars and young Planetary Nebulae. In these environments, $\mathrm{SiO}_{2} \mathrm{H}_{2} \mathrm{O}$ and $\mathrm{HCN}$ are molecules that can naturally generate polarized maser emission observable by ALMA. The maser polarization can then be used to derive the strength and morphology of the magnetic field in the masing regions. However, in order to derive, in particular, the magnetic field orientation from maser linear polarization, a number of conditions involving the rate of stimulated emission, molecular state decay and Zeeman splitting need to be satisfied. In this work, we discuss these conditions for the maser transitions in the ALMA frequency range and highlight the optimum transitions to further our understanding of star formation and evolved star magnetic fields.
\end{abstract}

Keywords. masers, polarization, stars: magnetic fields, stars: AGB and post-AGB, stars: formation.

\section{Introduction}

Polarized maser emission has been detected towards Star Forming Regions (SFR) and expanding Circumstellar Envelopes (CSE) of late-type stars like (post-) Asymptotic Giant Branch (AGB) stars and young Planetary Nebulae (PNe). Both single dish and interferometric observations have revealed that Silicon monoxide $(\mathrm{SiO})$, Water $\left(\mathrm{H}_{2} \mathrm{O}\right)$, Hydrogen cyanide $(\mathrm{HCN})$, Hydroxyl $(\mathrm{OH})$ and Methanol $\left(\mathrm{CH}_{3} \mathrm{OH}\right)$, among others; can naturally generate polarized maser emission in such enviroments (e.g. Vlemmings et al 2006). According to the Zeeman interpretation, the character of the polarization depends strongly on the ratio between the Zeeman frequency $(\mathrm{g} \Omega)$, the rate of stimulated emission $(\mathrm{R})$ and the rate of the decay of the molecular state $(\Gamma)$. The Zeeman splitting induced by a magnetic field depends on the molecule's shell structure. Since $\mathrm{SiO}, \mathrm{H}_{2} \mathrm{O}$ and $\mathrm{HCN}$ are non-paramagnetic, closed-shell molecules; $\mathrm{g} \Omega$ is expected to be less than the intrinsic line breadth $\Delta \omega$. In this case, the fractional polarization $\left(p_{L}\right)$ is typically of order a few percent. To explain the high levels of fractional linear polarization detected in some cases (specifically $\mathrm{SiO}$ masers, e.g. Amiri et al 2012, Vlemmings et al. 2011) other physical processes that can produce polarized maser radiation need to be considered. Anisotropic pumping can, for example, enhance the fractional linear polarized maser radiation that is produced due to a magnetic field permeating the masing region, towards SFR or CSE of late-type stars (Vlemmings et al. 2011, Nedoluha \& Watson 1990).

The different conditions (temperature and gas density) required by each molecular specie to generate polarized maser emission, allow us to constrain the magnetic field properties, i.e., field strength and/or direction, in different regions around proto-stars or 
Table 1. In the ALMA frequency range a number of $\mathrm{SiO}$ maser transitions, of up to $J=7-6$ for vibrational-excited states of up to $\nu=3$, could be detected. Here we list some of the most relevant ones detected mostly towards CSE of late-type stars, including the ALMA band where the different lines could be observed.

\begin{tabular}{c|c|c|c}
\hline & $\mathrm{SiO}$ & ${ }^{29} \mathrm{SiO}$ & ${ }^{30} \mathrm{SiO}$ \\
\hline & $\mathrm{GHz} ; \mathrm{Band}$ & $\mathrm{GHz} ; \mathrm{Band}$ & $\mathrm{GHz}$ Band \\
\hline$\nu=1$ & $43.12(\mathrm{~J}=1-0) ; \mathrm{B} 1$ & $127.74(\mathrm{~J}=3-2) ; \mathrm{B} 4$ & $168.32(\mathrm{~J}=4-3) ; \mathrm{B} 5$ \\
& $86.24(\mathrm{~J}=2-1) ; \mathrm{B} 2$ & $170.32(\mathrm{~J}=4-3) ; \mathrm{B} 5$ & \\
& $301.81(\mathrm{~J}=7-6) ; \mathrm{B} 7$ & $253.70(\mathrm{~J}=6-5) ; \mathrm{B} 6$ & \\
\hline$\nu=2$ & $128.45(\mathrm{~J}=3-2) ; \mathrm{B} 4$ & $255.47(\mathrm{~J}=6-5) ; \mathrm{B} 6$ & $167.16(\mathrm{~J}=4-3) ; \mathrm{B} 5$ \\
& $171.27(\mathrm{~J}=4-3) ; \mathrm{B} 5$ & & \\
& $214.08(\mathrm{~J}=5-4) ; \mathrm{B} 6$ & & \\
\hline \multirow{2}{*}{$\nu=3$} & $127.55(\mathrm{~J}=3-2) ; \mathrm{B} 4$ & & \\
& $170.07(\mathrm{~J}=4-3) ; \mathrm{B} 5$ & & \\
& $212.58(\mathrm{~J}=5-4) ; \mathrm{B} 6$ & & \\
\hline
\end{tabular}

in CSEs. Interferometric observations at radio wavelengths have become a useful tool to perform a deeper study of polarized maser emission towards SFR and late-type stars. The new generation of observatories and instruments enable the study of maser radiation from higher vibrational-excited rotational transition with better sensitivity and resolution. Nowadays, The Atacama Large (sub-) Millimeter Array (ALMA) is starting to perform early science observations. Gradually all of its capabilities including polarimetry will be available for full science observations. In the ALMA frequency range a number of $\mathrm{SiO}, \mathrm{H}_{2} \mathrm{O}$ and $\mathrm{HCN}$ maser transitions belonging to vibrational-excited levels of up to $\nu=3$ can be observed (Table 1 and 2). Maser polarization theory (e.g. Western \& Watson 1984) predict fractional linear polarization levels of up to $100 \%$ for $J=1-0$ rotational transitions of diatomic molecules. Such polarization levels can be reached considering the presence of a magnetic field of a few Gauss in, for example, the $\mathrm{SiO}$ masing region in the CSE of late-type stars. But in the case of higher rotational transitions (i.e. $J=2-1$, $J=3-2$, etc.) theory also predicts that the fractional linear polarization should decrease as the angular momentum number of the involved state increases. Using Submillimeter Array (SMA) observations, Vlemmings et al. (2011) have measured fractional linear polarization of the $\nu=1, J=5-4 \mathrm{SiO}$ masers of VX Sgr of up to $\sim 40 \%$. In the Zeeman interpretation frame, such high fractional polarization levels need very strong magnetic fields in the masing region in order to be generated, which results in an unlikely strong magnetic field in the surface of the stars. However, the fractional linear polarization level can increase with the angular momentum number if anisotropic pumping is considered. Thus, the problem turns into determining if the fractional linear polarization measured for those molecular states still can be used to trace the magnetic field structure in the masing region. In order to determine that, it is necessary to evaluate the ratios between the maser parameters $\mathrm{R}, g \Omega$ and $\Gamma$ for each single rotational transition detected with angular momentum higher than $J=1$. The main goal of this work is to determine if the polarized maser radiation produced by molecular transitions of $\mathrm{SiO}, \mathrm{H}_{2} \mathrm{O}$ and $\mathrm{HCN}$ in the ALMA frequency range, could generate suitable levels of linear fractional polarization to study the magnetic field structure towards SFR and CSE of (post-) AGB stars. To do this, we have run numerical models adapted from Nedoluha \& Watson (1992) in order to calculate the fractional linear polarization level that could be generated by the 
Table 2. A few of both the $\mathrm{H}_{2} \mathrm{O}$ and $\mathrm{HCN}$ maser lines that will be observed in the ALMA frequency range.

\begin{tabular}{|c|c|c|c|c|c|}
\hline & $\mathrm{H}_{2} \mathrm{O}$ & & & $\mathrm{HCN}$ & \\
\hline $\mathrm{J}_{K_{a}, K_{c}}$ & GHz; Band & Source type & & GHz; Band & Source type \\
\hline $\begin{array}{l}3_{30}-2_{20} \\
5_{15}-4_{22} \\
6_{43}-5_{50}\end{array}$ & $\begin{array}{l}183.31 ; \mathrm{B} 5 \\
325.15 ; \mathrm{B} 7 \\
439.15 ; \mathrm{B} 8\end{array}$ & $\begin{array}{l}\text { SFR, O-CSE } \\
\text { SFR, O-CSE } \\
\text { SFR, O-CSE }\end{array}$ & $\begin{array}{c}\mathrm{J}=1-0 \\
\nu_{2}=1^{1} \\
\mathrm{~J}=2-1 \\
\mathrm{~J}=3-2 \\
\mathrm{~J}=4-3\end{array}$ & $\begin{array}{l}177.2 ; \mathrm{B} 5 \\
267.2 ; \mathrm{B} 6 \\
354.5 ; \mathrm{B} 7\end{array}$ & $\begin{array}{l}\text { C-CSE } \\
\text { C-CSE } \\
\text { C-CSE } \\
\text { C-CSE }\end{array}$ \\
\hline $\begin{array}{c}\nu_{2}=2 \\
1_{10}-1_{01}\end{array}$ & $658.01 ; \mathrm{B} 9$ & O-CSE & & & \\
\hline
\end{tabular}

interaction of the molecular states of $\mathrm{SiO}, \mathrm{H}_{2} \mathrm{O}$ and $\mathrm{HCN}$ in the ALMA frequency range, with a magnetic field in the masing region.

\section{Overview}

Low frequency observations of maser emission from astrophysical sources have been shown to be excellent tools to study the magnetic field structure towards SFR and latetype stars. As mentioned above, the polarization characteristics of the maser emission strongly depend on the radiative conditions of the region where the maser radiation is being generate (saturated or unsaturated). Saturated maser is defined as the radiative regime where the stimulated emission rate $R$ overcomes the decay rate, $\Gamma$. In this case, the growth of the possible polarization modes is determined by the population of the molecular states which can interact with a particular mode of polarization (Nedoluha \& Watson 1990). Maser polarized emission can be produced in both the unsaturated and the saturated regimes. Goldreich et al. (1973) first identified two regimes in the saturated frame where, in the presence of a magnetic field, polarized radiation could be generated: a) The strong magnetic field regime, $g \Omega \gg \mathrm{R}$; b) The intermediate strength magnetic field regime, $(g \Omega)^{2} / \Gamma \gg \mathrm{R} \gg g \Omega$, for angular molecular transition $\mathrm{J}=1-0$. Watson and co-workers (Western \& Watson 1984, Deguchi \& Watson 1990, Nedoluha \& Watson 1990) did extend this treatment solving the radiative transfer equations for polarized maser radiation as a function of the emergent intensity $\mathrm{R} / \Gamma$, taking into account molecular transitions of up to $J=3-2$. Nedoluha \& Watson (1990) have shown that $p_{L}$ decreases when the rotational transition involved has molecular levels with higher angular momentum, and varies as a function of the angle $(\theta)$ between the magnetic field lines $(\vec{B})$ and the direction of propagation of the maser radiation $(\vec{k})$. When the magnetic field is perpendicular to the propagation direction of the maser radiation, the fractional linear polarization reaches its maximum value, decreasing to its minimum value when $\theta$ comes close to zero.

The angle that the vector of polarization makes with the $\vec{k} \cdot \vec{B}$ plane, $\phi$, changes as a function of the ratio $g \Omega / \mathrm{R}$ (Nedoluha \& Watson 1990). For a fixed value of $\theta$, the vector of polarization should be either parallel or perpendicular to the $k B$ plane if the Zeeman frequency overcomes the stimulated emission rate, $g \Omega>\mathrm{R}$. In the other hand, for larger values of $\mathrm{R}$, but still in the intermediate strength magnetic field regime, the vector of polarization is already rotated away from the $k B$ plane, ie, if $\mathrm{R}>g \Omega$ the vector of polarization is neither parallel nor perpedicular to the $k B$ plane. Therefore, 
as long as the condition $g \Omega>\mathrm{R}$ is satisfied, the information about the morphology of the magnetic field in the masing region could be extracted from the polarization vector.

Observationally, fractional linear polarization of up to $100 \%$ for $\mathrm{J}=1-0 \mathrm{SiO}$ maser transitions (Amiri et al. 2012) and values over 33\% for $\mathrm{SiO}$ molecular transitions involving higher angular momentum states (e.g. Vlemmings et al. 2011) have been detected. Anisotropic pumping seems to be a plausible explanation for such high $p_{L}$ values (Nedoluha \& Watson 1990). Contrary to the effect of a magnetic field alone in the masing region, the linear polarization can increase with the angular momentum of the involved states due to the anisotropic pumping. But even considering the linear polarization has been enhanced by anisotropic pumping, if $g \Omega>\mathrm{R}$, the magnetic field structure still can be traced directly from the polarization vector.

\section{Results}

The goal of this work is to determine if the different maser transitions in the ALMA frequency range can generate reliable levels of fractional linear polarization in the strong magnetic field regime. In order to evaluate whether $\mathrm{R}$ satisfied the condition $g \Omega \gg \mathrm{R}$, we have used a radiative transfer code adapted from Nedoluha \& Watson (1992). Since $\mathrm{R}$ cannot be determined directly from the observations, it has been calculated using:

$$
R \approx \frac{A k T_{b} \Delta \Omega}{4 \pi \hbar \omega}
$$

where $\mathrm{A}$ is the Einstein coefficient of the involved transition, $\mathrm{k}$ the boltzmann constant, $\mathrm{T}_{b}$ the brightness temperature and $\Delta \Omega$ the beaming solid angle for the maser radiation. From the observations, it is possible to estimate the value of $\mathrm{T}_{b}$, but it is not straightforward to determine the value of $\Delta \Omega$.

Here, we present partial numerical results of our models (Perez-Sanchez \& Vlemmings 2012 , in prep). In Fig 1 we present the results for the $325 \mathrm{GHz}, 5_{15}-4_{22} \mathrm{H}_{2} \mathrm{O}$ maser transition, as well as for the $215 \mathrm{GHz}, \nu=1, J=5-4 \mathrm{SiO}$ maser line.

\section{Conclusions}

From our models for $\mathrm{H}_{2} \mathrm{O}$ and $\mathrm{SiO}$, it seems likely that polarized maser emission lines in the ALMA frequency range can be used in order of determining the magnetic field structure towards late-type stars and SFR. In Figure 1 it is clear that, in the case of SiO, suitable levels of fractional linear polarization, even in the case of $\theta>75$ degrees, could be observed in the strong magnetic field regime $g \Omega>\mathrm{R}$. In our models, we have used typical magnetic field strength reported in the literature for both the $\mathrm{H}_{2} \mathrm{O}(50 \mathrm{mG})$ and the $\mathrm{SiO}$ (1 G) masing regions (Nedoluha \& Watson 1990, Nedoluha \& Watson 1992). Thus, still in the presence of anisotropic pumping, the polarization vector will trace the magnetic field, being either parallel or perpendicular to the direction of magnetic field in the plane of the sky. This agrees pretty well with what Vlemmings et al. (2011) suggested about tracing the magnetic field around VX sgr using the vector of polarization of the $\nu=1, J=5-4$ maser line. The uncertainties or our results are on the beaming angle of the maser radiation, which cannot be derived from observations. In the case of water, it seems that up to $10 \%$ of fractional linear polarization could be detected having $g \Omega>\mathrm{R}$. A complete analysis, including other maser lines of up to $\nu=3$ in the case of $\mathrm{SiO}$, is going to be submitted soon (Perez-Sanchez \& Vlemmings 2012, in prep). 

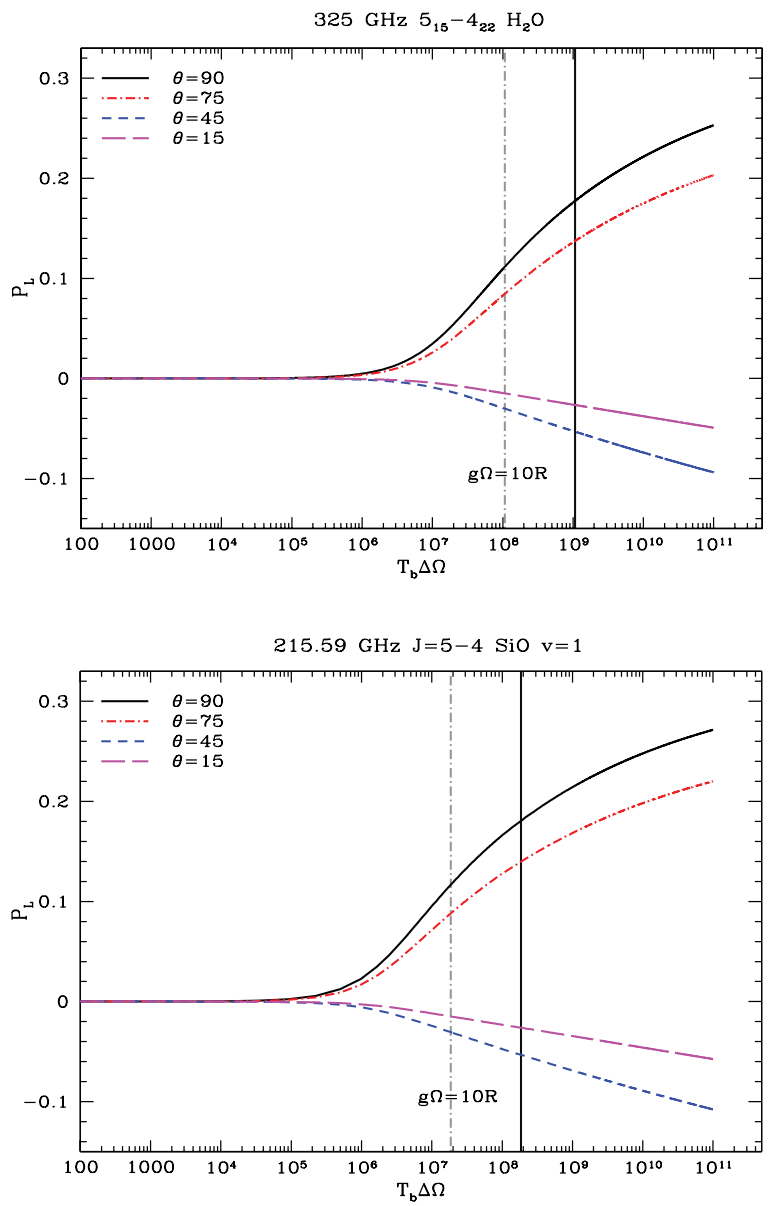

Figure 1. Fractional linear polarization as a function of $\mathrm{T}_{b} \Delta \Omega$ for two representative lines of $\mathrm{H}_{2} \mathrm{O}$ and $\mathrm{SiO}$ masers in the ALMA frequency range, for four different values of $\theta(90,75,45$, 15 degrees). The gray line points out the value of $\mathrm{T}_{b} \Delta \Omega$ where polarization should be reliable observed for each line, whereas the black one shows the limit value where $\mathrm{g} \Omega=\mathrm{R}$. For water we have considered a magnetic field strength of $50 \mathrm{mG}$ and decay rate of $\Gamma=1 \mathrm{~s}^{-1}$. Taking into account a beaming solid angle of $\Delta \Omega=10^{-2}$, water masers with brigthness temperature of a few $\times 10^{10} \mathrm{~K}$ can generate at least $10 \%$ of fractional linear polarization in the presence of a magnetic field perpendicular to the direction of propagation of the maser radiation. In the case of $\mathrm{SiO}$, considering $|B|=1 G$ and $\Gamma=5 s^{-2}$, and once again taking $\Delta \Omega=10^{-2}$, it seems likely to observe about $10 \%$ of fractional linear polarization due to the action of a magnetic field, still satisfying the inequality $g \Omega>\mathrm{R}$.

\section{References}

Amiri, N., Vlemmings, W. H. T., Kemball, A. J., \& van Langevelde, H. J. 2012, A\&\&A, 538, A136

Deguchi, S. \& Watson, W. D. 1990, ApJ, 354, 649

Goldreich, P., Keeley, D. A., \& Kwan, J. Y. 1973, ApJ, 179, 111

Nedoluha, G. E. \& Watson, W. D. 1990, ApJ, 354, 660

Nedoluha, G. E. \& Watson, W. D. 1992, ApJ, 384, 185

Perez-Sanchez, A. F. \& Vlemmings, W. 2012, in preparation

Vlemmings, W. H. T., Diamond, P. J., \& Imai, H. 2006, Nature, 440, 58

Vlemmings, W. H. T., Humphreys, E. M. L., \& Franco-Hernández, R. 2011, ApJ, 728, 149

Western, L. R. \& Watson, W. D. 1984, ApJ, 285, 158 